\title{
The effect of smoking on sensory and attentional masking
}

\author{
PAUL ATCHLEY, JAMES GROBE, and LACY M. FIELDS \\ University of Kansas, Lawrence, Kansas
}

\begin{abstract}
This study examined the influence of smoking on low-level sensory and higher level visual cognitive abilities. Three groups (nonsmokers, deprived smokers, and nondeprived smokers) of 22 observers were tested using a visual target discrimination task. To assess sensory and visual cognitive differences, a masking task developed by Enns and Di Lollo (1997) was used. In the task, the target was masked by either a contour mask (contour masking) or an object mask (attentional masking by object substitution). The strongest masking effects were found in the group of nondeprived smokers, for both sensory and attentional masking. This pattern of results as well as results in the deprived smokers indicated changes in transient visual processing channels due to the acute actions of nicotine, not mediated by withdrawal relief.
\end{abstract}

The purpose of this study was to examine the effect of cigarette smoking on low-level visual perception and related visual cognitive performance. One question in the high rate of relapse in smoking cessation attempts (over $90 \%$ by some accounts; Hughes, Higgins, \& Hatsukami, 1990 ) is the contribution of nicotine's enhancement of sensory and cognitive processing and the impairment in functioning seen during nicotine withdrawal. While it is generally recognized that the psychoactive properties of the nicotine present in cigarettes make it a drug of dependence (U.S. Department of Health and Human Services, 1988), smoking also produces demonstrable psychopharmacological benefits that may contribute to drug dependence. For example, if nicotine enhances sustained attention, producing a perception of increased mental alertness, smoking behavior could be reinforced (Warburton, 1990; Warburton, Revell, \& Walters, 1988; Wesnes \& Warburton, 1983). Understanding the spectrum of changes to sensory and cognitive processes that are produced by smoking is an important step toward the development of intervention strategies for smoking cessation.

Although some of the data are certainly mixed with regard to the influence of smoking and nicotine on the human performance across a wide range of measures, a preponderance of the evidence from studies with abstinent smokers indicates that smoking does have an effect on a variety of sensory, perceptual, motor, and cognitive tasks (Heishman, Taylor, \& Henningfield, 1994). Within the context of the classic information processing view of

\footnotetext{
We thank Stacia Miller for her assistance on the project and two anonymous reviewers for their helpful comments on an earlier version of this paper. This work was supported a grant from the University of Kansas General Research Fund to P.A. Correspondence should be addressed to P. Atchley, Department of Psychology, 426 Fraser Hall, University of Kansas, Lawrence, KS 66045 (e-mail: patchley@ ku.edu).
}

human cognition, understanding changes to processes of sensation, perception, attention memory, and response selection and output is critical for development of a formal model for nicotine-induced changes in human cognition. To date, many of the studies available have investigated these processes in isolation. What is also equally critical for development of a model is understanding the interactions among these processes. For example, though memory is a process unto itself, it is also modulated by attentional factors. Thus nicotine may enhance memory storage processes themselves or enhance the attentional resources that facilitate information extraction from the environment of the smoker, or both (Warburton, Rusted, \& Müller, 1992). Alternatively, abstinence from nicotine in the dependent smoker may lead to decreased attentional resources and provide a context for tobacco reinforcement via withdrawal relief (e.g., Hatsukami, Fletcher, Morgan, Keenan, \& Amble, 1989).

Another set of processes in which interactions are particularly important are found in the human visual system. Though the common concept of vision is "to see one must simply have open eyes," recent psychophysical and neurophysiological evidence demonstrates that our visual system is more heavily modulated by our limited visualattentional capacity than previously suspected. For example, studies of eye movements reveal that we have an immediate representation of only a limited number of objects (about four) in the visual world around us (Irwin, 1996). This is well demonstrated in studies of "change blindness," in which participants fail to notice large changes to a visual scene (such as the disappearance of a building) during continuous subsequent presentations of pictures (Pringle, Irwin, Kramer, \& Atchley, 2001; Rensink, O'Regan, \& Clark, 1997). Equally compelling evidence from neuroscience shows that the response of neurons in the visual system to information in the visual field is highly dependent upon attention. For example, a 
neuron whose receptive field is tuned to respond to a particular motion responds at a lower, potentially subthreshold, rate when attention is allocated to a different location, even if the motion stimulus is still present in the appropriate receptive field (Treue \& Maunsell, 1996).

Visual perception is critical for the extraction of various sources of information in the world around us that might regulate smoking behavior. Understanding how smoking and nicotine influence these perceptual processes is important for developing models that predict how smokers extract information that regulates smoking behavior. There are a variety of sensory and perceptual abilities that might be examined. However, the choices are limited to an extent because, to examine this interaction, one must utilize tasks in which sensory processes can be disambiguated from attentional processes. Further, if the purpose is to predict the ability of nicotine to reinforce smoking by enhancement of perceptual abilities, it makes sense to begin with simple sensory abilities, which underlie more complex perceptual abilities.

Studies to date in this area have been primarily focused on critical flicker frequency (CFF) thresholds (Hindmarch, Kerr, \& Sherwood, 1990; Leigh, 1982; Sherwood, Kerr, \& Hindmarch, 1992; Waller \& Levander, 1980). Although results have been mixed, some studies (such as Waller \& Levander, 1980) have revealed small (on the order of $0.75 \mathrm{~Hz}$ ) reliable increases in CFF thresholds following tobacco smoking by tobacco-deprived smokers. However, it is unclear what changes in CFF reflect. In cited studies, changes in CFF are considered to reflect increases in central nervous system (CNS) arousal. For example, Waller and Levander noted that the effect on CFF produced by smoking is similar in time course to decreased alpha activity in EEG (Knott \& Venables, 1977), suggesting a cortical or central source of the effect. However, discrimination of flicker is accomplished through activity in the magnocellular system (Schiller, Logothetis, \& Charles, 1990, 1991). Thus, changes in precortical mechanisms, such as changes to the sensitivity of M-type retinal ganglion cells, could also account for changes in CFF. So, changes in CFF may reflect changes in cortical arousal, but they may also reflect changes at an early, sensory, stage, making CFF a less desirable candidate for examining sensory and cognitive interactions in smoking.

A better task to isolate sensory and cognitive processes is visual masking. Visual masking is the reduction in the visibility of one stimulus by the presentation of another stimulus in near or concurrent spatial and temporal proximity (Breitmeyer, 1984). There are various forms of visual masking. The form examined in this study is contour masking. ${ }^{1}$ In contour masking, the presence of a nearby but not spatially contiguous visual stimulus impairs recognition of a target stimulus. The example to consider is trying to read text in which the letters are placed too closely together. Reading in such a situation is slow and arduous, as perception of individual let- ters is degraded by other, nearby, letters. A reduction of this form of masking could be perceived, for example, as an increase in visual clarity. There are numerous explanations of the phenomenon; theories of contour masking presume that mechanisms underlying the phenomenon occur early in the visual processing stream (Breitmeyer \& Ganz, 1976; Burr, 1984; Weisstein, Ozog, \& Szoc, 1975). For example, the masking model of Breitmeyer and Ganz (1976; see also Breitmeyer, 1984; Breitmeyer \& Williams, 1990) accounts for masking by the interactions of activity in sustained (parvocellular) and transient (magnocellular) channels. Inhibitory activity both within channels and between channels contributes to masking.

To provide an appropriate attentional or cognitive analogue to a sensory masking manipulation, the present study relies upon the work of Enns and Di Lollo (1997). In their work, they compared contour masking to attentional masking by object substitution. To understand the difference, consider the two forms of masks depicted in Figure 1. The mask on the left is a typical contour mask. The surrounding frame crowds the target (the diamond) but does not overlap it. By comparison, the properties of the four-square mask on the right make it too weak to serve as a mask under typical conditions. However, when the target is presented with spatial uncertainty in such a way that attention must be divided across a large spatial extent in order to detect it, the set of squares on the right can produce masking (a reduction in the ability to accurately identify the target). Because this occurs only under conditions in which the attentional gradient is spread across a large spatial extent, Enns and Di Lollo have suggested that it is a form of attentional masking. The mechanism they have suggested is that the representation of the dots substitutes itself for the representation of the target (see also Brehaut, Enns, \& Di Lollo, 1999; Giesbrecht \& Di Lollo, 1998). Thus, whereas contour masking is a low-level, sensory, effect, object substitution is a higher order, cognitive, effect.
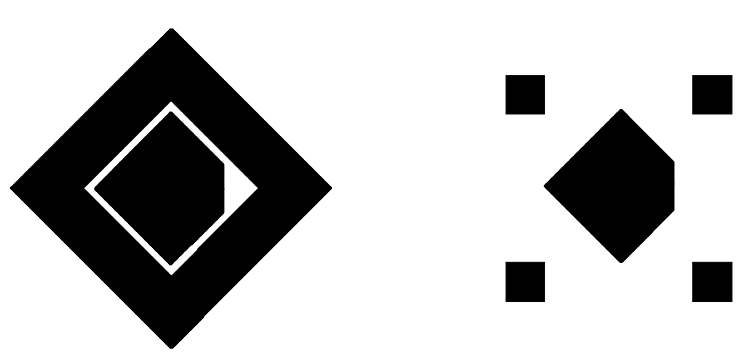

\section{Contour mask}

\section{Object mask}

Figure 1. Diagram of the two types of masks. The target is the center diamond. The contour mask on the left produces masking through metacontrast. The four-dot or object mask on the right produces masking through object substitution. 
How might the influence of smoking and nicotine interact with these effects? If increases in CFF that have been reported in smokers are the result of increases in magnocellular channel activity or sensitivity, then an increase in sensory or contour masking would also result. If the results of previous CFF studies can be interpreted to indicate changes in general CNS arousal, then it is predicted that smoking will positively impact attentional masking. The attentional masking effect is resource based (i.e., it occurs only when attention is spread across a large region). If the arousal produced by smoking leads to an increase in attentional resources (see Kassel, 1997, for a review and model), then smokers should enjoy less attentional masking. If, however, smoking produces only low-level sensory changes, then attentional masking should be the same for smokers, nonsmokers, and abstinent controls. However, since abstinence from nicotine in dependent smokers may result in impairments in these processes, the present study included nondeprived smokers and nonsmokers as one way to address such mechanisms (Hughes, 1991).

\section{METHOD}

\section{Participants}

Participants were 75 undergraduate students (25 in each smoking condition) from the University of Kansas. Nonsmokers (13 men and 12 women) were on average $19.08 \pm 1.22$ years old, denied current use of tobacco and reported smoking no more than 20 cigarettes in their lifetime. Smokers (15 men and 10 women) were on average $19.28 \pm 0.89$ years old, reported currently smoking $14.86 \pm 4.58$ cigarettes per day, scored $3.2 \pm 1.87$ on the Fagerstrom Test for Nicotine Dependence (FTND; scaled 0-10; $0=$ no measurable addiction, $10=$ highest level of addiction measured by the test; Fagerstrom, 1978; Heatherton, Kozlowski, Frecker, \& Fagerstrom, 1991 ), and had used tobacco for $3.82 \pm 1.58$ years. Deprived smokers (13 men and 12 women), who were on average $19.36 \pm 1.50$ years old, reported currently smoking $14.83 \pm 5.16$ cigarettes per day, scored $3.24 \pm 1.48$ on the FTND, and had used tobacco for $3.52 \pm 1.58$ years. (An analysis of variance revealed no significant age differences between groups and no significant differences in nicotine dependence between the deprived and nondeprived smokers.) ${ }^{2}$ The students were participating to obtain psychology credit, and the deprived smokers were also paid (\$5 each) for abstaining from nicotine. The protocol for human participants was approved by an institutional review board, and informed consent was obtained from all participants.

\section{Materials}

There were three types of stimuli in the masking task. The stimuli were derived from those used by Enns and Di Lollo (1997). All stimuli were black $\left(0.01 \mathrm{~cd} / \mathrm{m}^{2}\right)$ on a white background $\left(82.5 \mathrm{~cd} / \mathrm{m}^{2}\right)$. All stimuli were presented in a darkened room using a personal computer with a $45-\mathrm{cm}$ monitor set at a distance of $60 \mathrm{~cm}$. Observers were dark adapted for approximately 5-7 min while they were instructed on the experiment and given a brief demonstration. The target consisted of a diamond $\left(0.62^{\circ}\right.$ in vertical extent $)$ with a missing point $\left(0.17^{\circ}\right)$ on either the left or right side. The contour mask consisted of a diamond-shaped frame that fit around the target $\left(0.20^{\circ}\right.$ in width and 1 pixel from the target). The object mask consisted of four squares $\left(0.20^{\circ}\right)$ that were placed on a notional square $\left(1.0^{\circ}\right.$ on each side). The squares had a minimum separation from the target of $0.35^{\circ}$. Enns and Di Lollo found that these squares did not serve as a mask when viewed centrally (see also Breitmeyer, 1984; Growney, Weisstein, \& Cox, 1977). In addition to the primary stimuli, a fixation point consisting of two short vertical lines $\left(2.0^{\circ}\right.$ above and below the location of the central stimulus) was used.

There were three blocks of displays in the experiment. In the first display block, the target appeared alone in the central condition. The purpose of this block was to familiarize the participants with the identification task. In the second display block, the target and mask (contour and object masks appeared equally often and were randomly assigned from trial to trial) were presented in the central location only. In the third display block, the target and mask were randomly assigned one of three locations: one central and two parafoveal (3.0 left and right of center). All target and mask locations were used. Thus, the target and the mask appeared in the same location only on one third of the trials. Across all trials, the duration of the target and mask was $32 \mathrm{msec}$. The mask, when present, was presented at one of eight stimulus onset asynchronies (SOAs) $(-150,-100,-50,0,50,100,150 \text {, or } 300 \mathrm{msec} \text { relative to target })^{3}$ Within a block, there were 36 trials for display type 1, 160 trials for display type 2 (10 trials per SOA and mask type), and 288 trials for display type 3 . (When both the target and mask were co-located, there were 2 and 4 trials per SOA and mask type for the central and peripheral conditions, respectively).

\section{Procedure}

Prior to scheduling, participants were called for verification of their qualifications. Qualifications for smokers included smoking at a current rate of 10 or more cigarettes/day (on average), smoking at their current rate for at least 6 months, and being a regular (daily) smoker for at least 1 year. Qualifications for nonsmokers included smoking less than 20 cigarettes in their lifetime. Smokers were randomly assigned to either the deprived or smoking condition. The deprived smokers were instructed to not smoke for $10 \mathrm{~h}$ before their session, and the smokers were instructed to smoke at their normal rate up until the session. Upon arrival at the experiment, informed consent was obtained and the participant was escorted outside. All participants remained outside for 5-7 min. The smokers smoked a cigarette of the brand of their choice. The deprived smokers and nonsmokers were instructed to take deep breaths, as if taking a drag of a cigarette, approximately every $30 \mathrm{sec}$. This procedure was followed to equate the breathing activity of nonsmokers and deprived smokers with that of smokers. Once back in the lab, expired-air carbon monoxide was assessed to verify differences in tobacco exposure. A CO cutoff of $13 \mathrm{ppm}$ was used to confirm the abstinence from tobacco of nonsmokers and deprived smokers. Participants then completed some brief subjective measures and a brief task for another study (Miller \& Grobe, 2001).

In the masking task, participants were instructed that they would be performing a target identification task. They were informed that their task would be to determine whether a briefly presented diamond shape was missing its left or right corner. They were told to press the mouse button corresponding to the missing corner (left or right). They were also informed that the display would be very brief and occasionally made more difficult by the presence of competing visual information and were asked to respond as accurately as possible, to not worry about response speed, and to make their best guess when unsure of the correct answer. Observers were instructed to fixate between the vertical lines that appeared at the beginning of the trial. After these instructions, the observers were allowed to complete the first block of trials, in which they detected the target alone. After the first block, observers were informed that on subsequent blocks, trials would contain a mask that might occur before or after the target and that this mask might make the target difficult to detect. Observers were informed that on the next (second) block of trials, the mask and target would appear centrally (at the fixation 
location). But, in the final (third) block of trials, the mask and target might appear centrally or in peripheral visual locations, and the mask and target might not appear in the same location. Observers were informed to maintain fixation centrally and not shift gaze to the mask location, since it would not predict the location of the target with more than chance accuracy. After these instructions, observers completed one block of Condition 2 and two blocks of Condition 3 . Following completion of the study (approximately $30 \mathrm{~min}$ ), participants were debriefed and given their appropriate compensation.

\section{RESULTS}

Analyses of $\mathrm{CO}$ readings confirmed that groups significantly differed with respect to recent tobacco exposure $[F(1,72)=72.7, p<.001)$. Nondeprived smokers' $\mathrm{CO}$ readings were significantly higher than deprived smokers' and nonsmokers' CO readings (18.6 \pm 8.2 , $5.0 \pm 2.9,2.5 \pm 1.1$, respectively). Nonsmokers' CO readings were not significantly different from deprived smokers'. This confirms that manipulation of smoking status was successful.

During the initial data analysis, incomplete data sets were noted for 7 observers ( 2 nonsmokers, 3 deprived smokers, and 2 nondeprived smokers). Data from 2 additional observers were randomly excluded from analysis to equate the sample size among groups, resulting in 22 observers per group. In the central condition (second display block), there were 220 trials per data point, per group. In the three-location condition (third display block, which was run twice), for the same location condition, there were 4 and 8 trials for the central and peripheral cases for each SOA (producing 88 and 176 trials per data point per group, respectively). For the different location condition, there were 8 and 16 trials for the central and peripheral cases for each SOA (producing 176 and 352 trials per data point, respectively). The analysis was conducted by someone naive regarding the smoking status of the three groups.

\section{Central Condition Only}

Data from the central condition only (second display type) are presented in Figure 2 for the three observer types. Previous research (Enns \& Di Lollo, 1997) would predict a pattern of data in which there was no evidence of masking for the object mask across all SOAs and large masking for the contour mask at $50 \mathrm{msec}$. Moderate evidence of masking for both object and contour masks was found across a range of SOAs. To assess the degree of masking, an effect size analysis was conducted. In this analysis, the data from the SOAs of $<0 \mathrm{msec}$ were collapsed and used as a baseline to which the effect of masking could be compared. Previous research (Enns \& Di Lollo, 1997) failed to show consistent paracontrast or forward masking effects (masking of target information by previously presented information) in this condition. Although there was some evidence of small forward masking effects across some combinations of variables, the establishment of a baseline with the SOAs of $<0 \mathrm{msec}$ was necessary for the analysis.

Effect size of masking at the critical 50-msec SOA and for each mask type across the three observer groups was conducted using Cohen's $d$ (Cohen, 1988). Effect sizes of around 0.3 are considered small, 0.5 medium, and 0.8 large. The analysis revealed the following. First, the object mask produced, at best, only medium-sized masking effects. This was generally consistent across smoking status (nonsmoker $d=0.53$; deprived smoker $d=$ 0.58 ; nondeprived smoker $d=0.56$ ). Effect size is not available from previous studies. Enns and Di Lollo (1997) predicted no masking in this condition, though an examination of their data (see their Figure 1b) reveals a trend that is qualitatively similar to the one reported here. Second, and most importantly for this study, the contour mask produced strong masking effects at the predicted 50-msec SOA. However, a strong masking effect was found only for the nondeprived smokers $(d=0.83)$. Deprived smokers and nonsmokers had only moderate masking effects at the same SOA $(d \mathrm{~s}=0.51$ and 0.56 , respectively).

\section{Three-Location Condition}

Baseline performance. Data for the two masking conditions are shown in Figure 3, for each of the smoking groups. Note that here the term masking is used to describe the type of mask present in the display, but for the baseline conditions the mask is at a different spatial location from the target. Except where noted, univariate
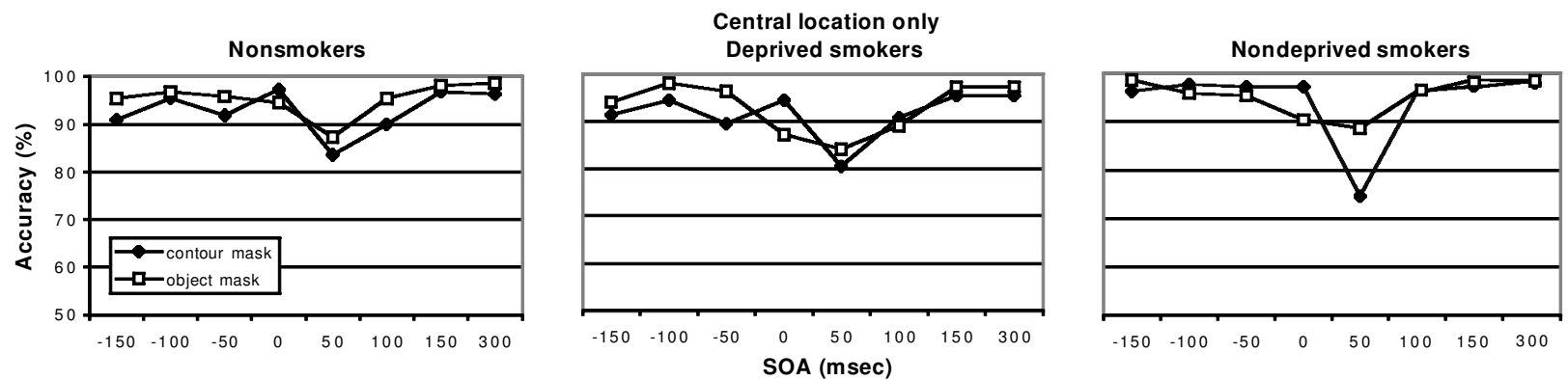

Figure 2. Effect of masking by contour and masking by object mask for the single target location condition. 

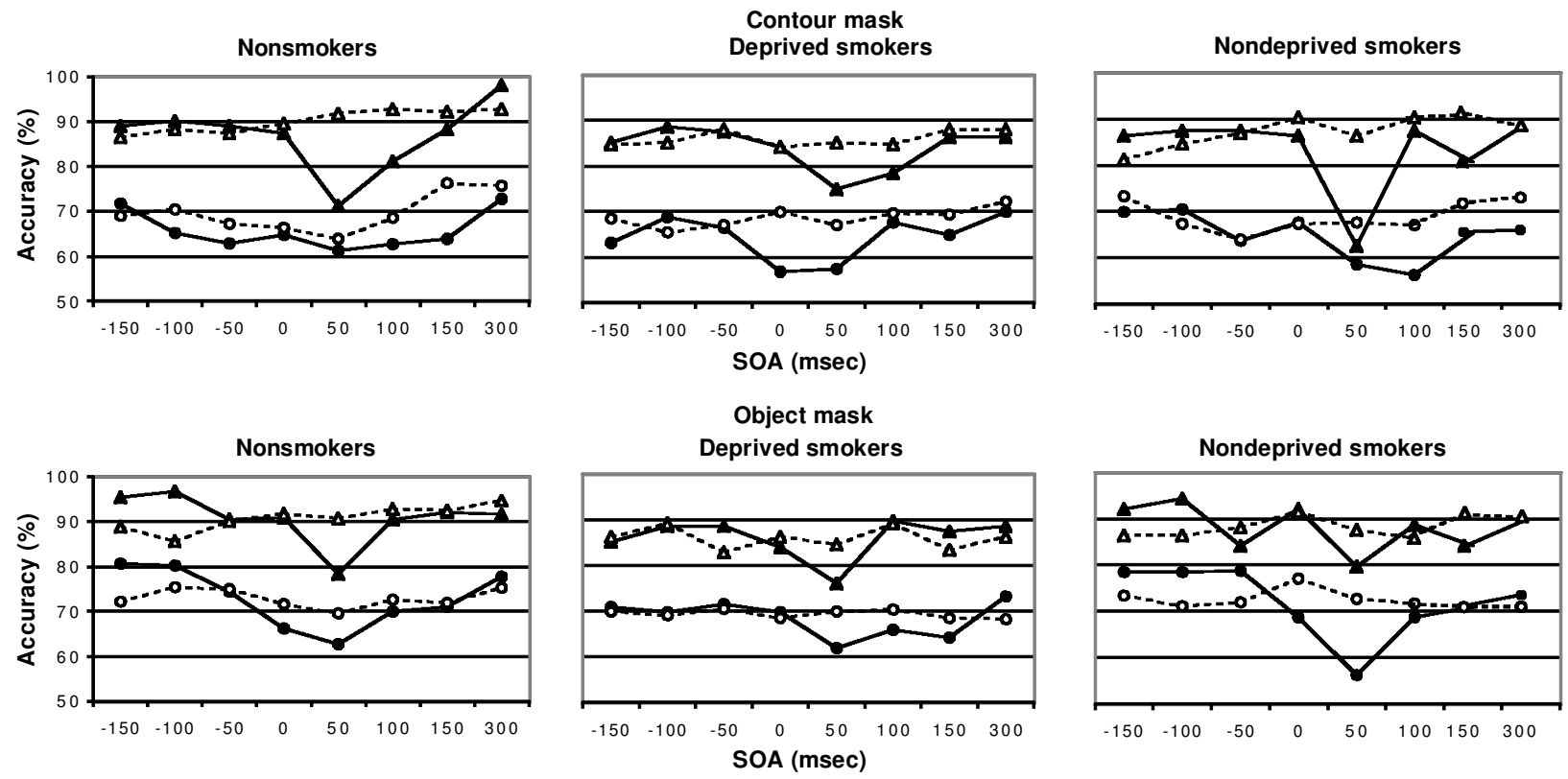

Figure 3. Masking in the three-location condition. The top set depicts performance with the contour mask and the bottom set performance with the object mask. Within each panel, the closed symbols show performance when the mask and target are in the same location and the open symbols show performance when the mask and target are in different locations. Triangles represent the central target location and circles the peripheral target location.

$F$ tests were used to test higher order interactions. As shown in Table 1, baseline performance (accuracy for a given location, central or peripheral, when the mask is at a different location) was similar across groups for central $\left[F(1,63)=0.82, M S_{\mathrm{e}}=2,177.2\right.$, n.s. $]$ and peripheral $\left[F(1,63)=0.28, M S_{\mathrm{e}}=1,859.2\right.$, n.s. $]$ locations. Baseline performance is better in the central target location than in the peripheral location $\left[F(1,63)=340.00, M S_{\mathrm{e}}=\right.$ $492.0, p<.01]$.

Forward masking and facilitation. Although there does not appear to be evidence for forward masking in any of the conditions, evidence of facilitation by the contour and object masks (better performance when the mask is present than when absent) occurred in some conditions. To conduct this analysis, negative SOA conditions were collapsed for both the baseline and masking conditions (target and mask in the same location). Each of these cases was compared for each smoking group, mask type, and target location. For the nonsmokers, facilitation was found for the object mask at both central $\left[F(1,63)=9.37, M S_{\mathrm{e}}=128.7, p<.01\right]$ and peripheral $\left[F(1,63)=4.00, M S_{\mathrm{e}}=148.5, p<.05\right]$ locations. No other effects were significant. There were no facilitory effects found in the deprived smoking group $\left(F_{\mathrm{S}}<1.0\right)$. In the nondeprived smoking group, facilitation was found for the object mask in the peripheral condition $\left[F(1,63)=9.18, M S_{\mathrm{e}}=148.5, p<.01\right]$. In the central condition, facilitation was found when the SOA range was restricted to -100 or less $\left[F(1,63)=8.50, M S_{\mathrm{e}}=120.9\right.$, $p<.01]$. No other effects were significant.

Contour and object masking. Effect size estimates (Cohen's $d$ ) were computed across the three groups for the contour and object masks for central and peripheral presentation. Performance for each condition was compared with the same performance in the baseline condition (when the target and distractor were in different locations). On the basis of previous research (Enns \& Di Lollo, 1997), it was expected that maximum masking should occur at about 50-msec SOA. All groups showed moderate contour masking (nonsmoker $d=0.55$; deprived smoker $d=0.46$; nondeprived smoker $d=0.66$ ) in central vision and small to moderate masking effects (nonsmoker $d=0.25$; deprived smoker $d=0.43$; nondeprived smoker $d=0.44$ ) in the parafoveal case, for the $50-\mathrm{msec}$

Table 1

Baseline Performance (\% Correct When Mask and Target in a Different Location) Across Group, Target Location, and Mask Type

\begin{tabular}{|c|c|c|c|c|c|c|}
\hline \multirow[b]{2}{*}{ Mask } & \multicolumn{2}{|c|}{ Nonsmokers } & \multicolumn{2}{|c|}{ Deprived Smokers } & \multicolumn{2}{|c|}{ Nondeprived Smokers } \\
\hline & Central & Peripheral & Central & Peripheral & Central & Peripheral \\
\hline Contour & 90.8 & 72.9 & 86.1 & 68.5 & 88.2 & 71.8 \\
\hline Object & 90.9 & 73.0 & 86.1 & 69.4 & 88.3 & 72.5 \\
\hline
\end{tabular}


SOA condition. In the object masking condition, all groups showed small to moderate masking in the central condition at $50-\mathrm{msec}$ SOA (nonsmoker $d=0.48$; deprived smoker $d=0.39$; nondeprived smoker $d=0.37$ ). In the peripheral condition, small to large masking effects were found at 50-msec SOA (nonsmoker $d=0.36$; deprived smoker $d=0.45$; nondeprived smoker $d=0.76$ ).

Since this condition is critical for assessment of attentional masking, it is important to account for the influence of sensory masking from these data. In other words, if the object mask produces sensory masking, then what effect remains when the sensory masking produced by the object mask is eliminated? Even when attention was not spread between multiple locations, the object mask produced a moderate amount of masking. This indicates that the attentional mask may also have a sensory masking component. However, the extent of this masking was equivalent across the three groups (nonsmoker $d=0.53$; deprived smoker $d=0.58$; nondeprived smoker $d=0.56$ ), whereas the effect size of the object mask at parafoveal locations in the three-location condition was not (nonsmoker $d=0.36$; deprived smoker $d=$ 0.45 ; nondeprived smoker $d=0.76$ ). Thus, it would appear that even if we consider that the attentional mask may have produced some degree of sensory masking, it is clear that additional, presumably attentional, masking occurs in at least the nondeprived smokers.

\section{DISCUSSION}

The purpose of this study was to examine perceptual and visual cognitive effects of smoking. The study utilized a masking methodology in which masking could occur due to perceptual factors (contour masking) or attentional masking (masking by object substitution) (Enns \& Di Lollo, 1997). The initial hypothesis was that the effect of nicotine provided by smoking could (1) increase sensory masking through increases transient channel activity and (2) enhance the attentional capabilities of the observers, resulting in decreased attentional masking. However, the data from this study were only consistent with the former conclusion. The nondeprived smokers in this study actually showed larger contour and attentional masking. Figure 2 indicates that in the central location only condition, all observers showed an effect for the contour mask at the predicted 50-msec SOA. However, this effect was more pronounced in the nondeprived smokers. An effect size analysis confirmed that nondeprived smokers had the largest masking effects. Thus, these results suggest that tobacco intake may directly alter visual processing. Attentional masking (masking by object substitution) was assessed by examining the effect of the object mask at peripheral locations in the three-location case, when attention is presumably spread across a larger spatial extent relative to the central only case. In this case, at the 50-msec SOA condition, nondeprived smokers showed the largest effect for the object mask, consistent with the general findings of the direct action of tobacco intake discussed above. The effect size of the mask was large for the nondeprived smokers, whereas they were small to moderate for the nonsmokers and deprived smokers, respectively.

Thus, one can conclude from the data that smoking has effects at both the visual sensory and visual cognitive levels, which did not appear to be mediated by withdrawal. This is supported by the large effect of the contour and object masks for the nondeprived smokers, effects that were smaller and/or absent in the nonsmoking and deprived smoking groups. With regard to contour masking, all models assume that the phenomenon is due to interactions among neurons early in visual processing (Breitmeyer \& Ganz, 1976; Burr, 1984; Weisstein et al., 1975). Therefore, the enhanced effects of the contour mask must reflect influences at low-level levels of visual sensory processing. Though the object mask may have produced weak sensory masking, when this was accounted for, the nondeprived smokers produced significant masking effects in the predicted condition. This indicates that the representation object mask substituted itself for the representation of the target (Brehaut et al., 1999; Enns \& Di Lollo, 1997; Giesbrecht \& Di Lollo, 1998).

What clues do we have to suggest a potential mechanism for these effects? One striking aspect of the data is that there were significant differences between the smoking groups with regard to the allocation of visual attention within the displays. First, in the central location only condition, only nonsmokers and deprived smokers showed forward masking. In other words, the presence of the mask before the target actually impaired performance. Smokers had equivalent performance at SOAs of $0 \mathrm{msec}$ or less. However, this is not the best case to consider, since nondeprived smokers had near ceiling performance at all SOAs of $0 \mathrm{msec}$ or less. A better condition to consider is the three-location condition, since cases within this condition include the necessary maskabsent (or mask-displaced) baseline for comparison. When visual attention was spread across the display, as would be required for performance in the three-location condition since the target location is randomized, nonsmokers and nondeprived smokers showed signs of facilitation with the object mask. When the object mask preceded the target, and the mask and target were in the same location, nonsmoker and nondeprived smoker performance was actually better than when the mask preceded the target and the mask and target were in different locations. (One would predict that the contour mask produced actual masking, which would nullify this benefit.) Although the effect of the object mask may reflect both a benefit (when the mask and target are in the same location) and a cost (when the mask and target are in different locations) for shifting attention, it does unequivocally indicate that these observers were shifting attention to the mask location.

Notably, observers knew a priori that the mask location would not predict the location of the target. There- 
fore, attentional orienting was a function of attentional capture by the mask onset (Theeuwes, Kramer, Hahn, \& Irwin, 1998; Yantis, 1996), rather than top-down control (Atchley, Kramer, \& Hillstrom, 2000; Folk, Remington, \& Johnston, 1992) or endogenous orienting of attention. Thus, it appears likely that the deprived smoking group showed a reduction in the orienting response. This provides another clue to the potential mechanism that withdrawal might have on the response of the deprived smokers. There is considerable evidence to suggest that activity in transient retinal channels are part of the mechanism of attentional capture by onsets, such as the onsets of the masks in this study (B. A. Steinman, S. B. Steinman, \& Lehmkuhle, 1995, 1997; S.B. Steinman, B. A. Steinman, Trick, \& Lehmkuhle, 1994), presumably, in part, due to direct projections of transient channels to the superior colliculus (Hoffman, 1973; Hoffman \& Sherman, 1975). Suppression of transient channel response could have the effect of decreasing the form of attentional orienting discussed here. This account is strengthened because changes in transient channel activity would also explain differences in masking performance.

As noted previously, the masking model of Breitmeyer and Ganz (1976; see also Breitmeyer, 1984; Breitmeyer \& Williams, 1990) accounts for masking through the interactions of activity in sustained and transient channels. Inhibitory activity both within channels and between channels contributes to masking. How is this related to increased masking found in the nondeprived smokers? Since the nondeprived smokers appeared to have an attentional orienting response to the onsets of the object mask similar to that of nonsmokers, one can assume that transient channel activity is intact in this group. (This also suggests that the notion that nicotine can aid with filtering "irrelevant" or "distracting" stimuli should be modified, see Grobe, Perkins, Goettler-Good, \& Wilson, 1998; Kassel, 1997). Yet, the effect of deprivation is to decrease the activity in these channels. The most likely model to account for this pattern of results consists of two processes. The first process is an increase in transient channel activity by smoking (and presumably the effect of nicotine). This would explain the increase in sensory masking with the smoking group, since transient on transient and transient on sustained channel inhibition would also increase with an overall increase in channel activity. The second process is the opponent process that occurs in response to this elevation of activity of the transient channels (e.g., Koob \& LeMoal, 1997). The result of deprivation (the lack of orienting in the deprived smokers) is a function of the second process, the opponent process decreasing activity in the transient channels, in the absence of the first process, the excitatory process engaged by smoking. (Attentional capture seems to be intact with the present observers.) This simple twoprocess model readily accounts for both the data from all three groups, including the effects of smoking and withdrawal from smoking.

To conclude, the proposal of modified transient channel activity having an impact on perceptual processing has recently been forwarded within a number of contexts. For example, the transient channel deficit model has been proposed to account for reading deficits (Breitmeyer, 1993; Lennerstrand, Ygge, \& Jacobsson, 1993; Slaghuis \& Ryan, 1999; B. A. Steinman, S. B. Steinman, Garzia, \& Lehmkuhle, 1996; but see also Iovino, Fletcher, Breitmeyer, \& Foorman, 1998). There is also the suggestion that transient channel changes may be associated with schizophrenia (Schwartz, Nelson, Wall, \& Winstead, 1985; Slaghuis, 1998; but see also Weiss, Chapman, Strauss, \& Gilmore, 1992). The selective transient channel influence by nicotine postulated here would seem to be consistent with explanations of other behavioral and cognitive changes. Thus, although nicotine may have some positive effects due to increases in attentional or perceptual resources, as others have postulated, the effects produced by changes to some sensory mechanisms may not be entirely without cost.

\section{REFERENCES}

Atchley, P., Kramer, A. F., \& Hillstrom, A. P. (2000). Contingent capture for onsets and offsets: Attentional set for perceptual transients. Journal of Experimental Psychology: Human Perception \& Performance, 26, 594-606.

Brehaut, J. C., Enns, J. T., \& Di Lollo, V. (1999).Visual masking plays two roles in the attentional blink. Perception \& Psychophysics, 61, 1436-1448.

Breitmeyer, B. G. (1984). Visual masking: An integrative approach. New York: Oxford University Press.

Breitmeyer, B. G. (1993). The roles of sustained (P) and transient (M) channels in reading and reading disability. In S. F. Wright \& R. Groner (Eds.), Facets of dyslexia and its remediation: Studies in visual information processing (Vol. 3, pp. 13-31). Amsterdam: Elsevier.

Brett meyer, B. G., \& GANZ, L. (1976). Implications of sustained and transient channels for theories of visual pattern masking, saccadic suppression, and information processing. Psychological Review, 82, 1-36.

Breitmeyer, B. G., \& Williams, M. C. (1990). Effects of isoluminantbackground color on metacontrast and stroboscopic motion: Interactions between sustained (P) and transient (M) channels. Vision Research, 30, 1069-1075.

Burr, D. C. (1984). Summation of target and mask metacontrast stimuli. Perception, 13, 183-192.

CoHen, J. (1988). Statistical power analysis for the behavioral sciences (2nd ed.). Hillsdale, NJ: Erlbaum.

EnNs, J. T., \& Di Lollo, V. (1997). Object substitution: A new form of masking in unattended visual locations. Psychological Science, 8, 135-139.

Fagerstrom, K. O. (1978). Measuring degree of physical dependence to tobacco smoking with reference to individualization of treatment. Addictive Behaviors, 3, 235-241.

Folk, C. L., Remington, R. W., \& Johnston, J. C. (1992). Involuntary covert orienting is contingent on attentional control settings. Journal of Experimental Psychology: Human Perception \& Performance, 18, 1030-1044.

Giesbrecht, B., \& Di Lollo, V. (1998). Beyond the attentional blink: Visual masking by object substitution. Journal of Experimental Psychology: Human Perception \& Performance, 24, 1454-1466.

Grobe, J. E., Perkins, K. A., Goettler-Good, J., \& Wilson, A. (1998). Importance of environmental context in the effects of nicotine on short-term memory. Experimental \& Clinical Psychopharmacology, 6, 209-216.

Growney, R., Weisstein, N., \& Cox, S. J. (1977). Metacontrast as a function of spatial separation with narrow line targets and masks. $\mathrm{Vi}$ sion Research, 17, 1205-1210.

Hatsukami, D., Fletcher, L., Morgan, S., Keenan, R., \& Amble, P. (1989). The effects of varying cigarette deprivation duration on cognitive and performance tasks. Journal of Substance Abuse, 1, 407-416. 
Heatherton, T. F., Kozlowski, L. T., Frecker, R. C., \& Fagerstrom, K. O. (1991). The Fagerstrom Test for nicotine dependence: A revision of the Fagerstrom Tolerance Questionnaire. British Journal of Addiction, 86, 1119-1127.

Heishman, S. J. (1998). What aspects of human performance are truly enhanced by nicotine? Addiction, 93, 317-320.

Heishman, S. J., Taylor, R. C., \& Henningfield, J. E. (1994). Nicotine and smoking: A review of effects on human performance. Experimental \& Clinical Psychopharmacology, 2, 345-395.

Hindmarch, I., Kerr, J. S., \& Sherwood, N. (1990). Effects of nicotine gum on psychomotor performance in smokers and nonsmokers. Psychopharmacology, 100, 535-541.

Hoffman, K. P. (1973). Conduction velocity in pathways from retina to superior colliculus in the cat: A correlation with receptive field properties. Journal of Neurophysiology, 36, 409-424.

Hoffman, K. P., \& Sherman, S. M. (1975). Early effects of binocular deprivation of visual input to cat superior colliculus. Journal of Neurophysiology, 38, 1049-1059.

Hughes, J. R., (1991). Distinguishing withdrawal relief and direct effects of smoking. Psychopharmacology, 104, 409-410.

Hughes, J. R., Higgins, S. T., \& Hatsukami, D. (1990). Effects of abstinence from tobacco: A critical review. In L. T. Koslowski, H. M. Annis, H. D. Cappell, F. B. Glaser, M. S. Goodstadt, Y. Israel, H. Kalant, E. M. Sellers, \& E. R. Vingilis (Eds.), Recent advances in alcohol and drug problems (Vol. 10, pp. 317-398). New York: Plenum.

Iovino, I., Fletcher, J. M., Breitmeyer, B. G., \& Foorman, B. R. (1998). Colored overlays for visual perceptual deficits in children with reading disability and attention deficit/hyperactivity disorder: Are they differentially effective? Journal of Clinical \& Experimental Neuropsychology, 20, 791-806.

IRwIN, D. E. (1996). Integrating information across saccadic eye movements. Current Directions in Psychological Science, 5, 94-100.

KASSEL, J. D. (1997). Smoking and attention: A review and reformulation of the stimulus-filter hypothesis. Clinical Psychology Review, 17, 451-478.

Knott, J. V., \& Venables, P. H. (1977). EEG alpha correlates of nonsmokers, smokers, smoking, and smoking deprivation. Psychophysiology, 14, 150-156.

Коов, G. F., \& LeMoal, M. (1997). Drug abuse: Hedonic homeostatic dysregulation. Science, 278, 52-58.

LEIGH, G. (1982). The combined effects of alcohol consumption and cigarette smoking on critical flicker frequency. Addictive Behaviors, 7, 251-259.

Lennerstrand, G., YGge, J., \& Jacobsson, C. (1993). Control of binocular eye movements in normals and dyslexics. In P. Tallal et al. (Eds.), Temporal information processing in the nervous system: Special reference to dyslexia and dysphasia (Annals of the New York Academy of Sciences, Vol. 682, pp. 231-239). New York: New York Academy of Sciences.

Miller, S., \& Grobe, J. (2001). The effects of smoking on visual sensory memory. Manuscript in preparation.

Perkins, K. A., Epstein, L. H., Grobe, J. E., \& Fonte, C. (1994). Tobacco abstinence smoking cues and the reinforcing value of smoking. Pharmacology, Biochemistry \& Behavior, 47, 107-112.

Pringle, H. L., Irwin, D. E., Kramer, A. F., \& Atchley, P. (2001). The role of attentional breadth in perceptual change detection. Psychonomic Bulletin \& Review, 8, 89-95.

Rensink, R. A., O'Regan, J. K., \& Clark, J. J. (1997). To see or not to see: The need for attention to perceive changes in scenes. Psychological Science, 8, 368-373.

Schiller, P. H., Logothetis, N. K., \& Charles, E. R. (1990). Role of the color-opponent and broad-band channels in vision. Visual Neuroscience, 5, 321-346.

Schiller, P. H., Logothetis, N. K., \& Charles, E. R. (1991). Parallel pathways in the visual system: Their role in perception at isoluminance. Neuropsychologia, 29, 433-441.

Schwartz, B. D., Nelson, A. V., Wall, M., \& Winstead, D. K. (1985). Visual evoked potentials associated with neural filter mechanisms in schizophrenia. New Trends in Experimental \& Clinical Psychiatry, 1, 179-185.
Sherwood, N., Kerr, J. S., \& Hindmarch, I. (1992). Psychomotor performance in smokers following single and repeated doses of nicotine gum. Psychopharmacology, 108, 432-436.

SlaghuIS, W. L. (1998). Contrast sensitivity for stationary and drifting spatial frequency gratings in positive- and negative-symptom schizophrenia. Journal of Abnormal Psychology, 107, 49-62.

Slaghuis, W. L., \& Ryan, J. F. (1999). Spatio-temporal contrast sensitivity, coherent motion and visible persistence in developmental dyslexia. Vision Research, 39, 651-668.

Steinman, B. A., Steinman, S. B., Garzia, R. P., \& Lehmkuhle, S. (1996). Vision and Reading III: Visual attention. Journal of Optometric Vision Development, 27, 4-28.

Steinman, B. A., Steinman, S. B., \& Lehmkuhle, S. (1995). Line motion illusion reveals that visual attention mechanisms have centersurround organization. Vision Research, 35, 1859-1869.

Steinman, B. A., Steinman, S. B., \& Lehmkuhle, S. (1997). Visual attention is dominated by the M-stream. Vision Research, 37, 17-23.

Steinman, S. B., Steinman, B. A., Trick, G. L., \& Lehmkuhle, S. (1994). A sensory explanation for visual attention deficits in the elderly. Optometry \& Visual Science, 71, 743-759.

Theeuwes, J., Kramer, A. F., Hahn, S., \& Irwin, D. E. (1998). Our eyes do not always go where we want them to go: Capture of the eyes by new objects. Psychological Science, 9, 379-385.

Treue, S., \& Maunsell, J. H. R. (1996). Attentional modulation of visual motion processing in cortical areas MT and MST. Nature, 382, 539-541.

U.S. Department of Health and Human Services. (1988). The health consequences of smoking: Nicotine addiction. Report of the Surgeon General. Washington, DC: U.S. Government Printing Office.

WALler, D., \& Levander, S. (1980). Smoking and vigilance: The effect of tobacco smoking on CFF as related to personality and smoking habits. Psychopharmacology, 70, 131-136.

Warburton, D. M. (1990). The pleasures of nicotine. In F. Adlkofer \& $\mathrm{K}$. Thurau (Eds.), Effects of nicotine on biological systems (pp. 473483). Basel: Birkhauser Verlag.

Warburton, D. M., Revell, A., \& Walters, A. C. (1988). Nicotine as a resource. In M. J. Rand \& K. Thurau (Eds.), The pharmacology of nicotine: Proceedings, Satellite Symposium of the Tenth International Congress of Pharmacology (pp. 359-373). Washington, DC: IRL Press.

Warburton, D. M., Rusted, J. M., \& Müller, C. (1992). A comparison of the attentional and consolidation hypotheses for the facilitation of memory by nicotine. Psychopharmacology, 3, 375-378.

Weiss, K. M., Chapman, H. A., Strauss, M. E., \& Gilmore, G. C. (1992). Visual information decoding deficits in schizophrenia. Psychiatry Research, 44, 203-216.

Weisstein, N. A., Ozog, G., \& Szoc, R. (1975). A comparison and elaboration of two models of metacontrast. Psychological Review, 82, 325-343.

WeSNEs, K., \& WARburton, D. M. (1983). Effects of smoking on rapid information processing. Neuropsychobiology, 9, 223-229.

YANTIS, S. (1996). Attentional capture in vision. In A. F. Kramer, M. G. G. Coles, \& G. D. Logan (Eds.), Converging operations in the study of visual selective attention (pp. 45-76). Washington, DC: American Psychological Association.

\section{NOTES}

1. Because this study, as well as the study by Enns and Di Lollo (1997), upon which the present stimuli are based, includes both negative and positive stimulus onset asynchronies (masks that occur before and after the target), there are actually two forms of masking: paracontrast masking, which is the term used for masking when masks occur before the target, and metacontrast masking, which is the term for masking when the mask occurs after the target (Breitmeyer, 1984).

2 . This smoking group methodology entails several considerations. First, the lack of a placebo cigarette group fails to control for other stimuli associated with smoking (Heishman, 1998). In addition, it is impossible to isolate effects of nicotine, per se, from those of tobacco smoking. Furthermore, lack of control over nicotine dosing prevents specifica- 
tion of dose-response relationships, which is particularly important for nicotine given the possibility of biphasic dose-response relationships (e.g., Perkins, Epstein, Grobe, \& Fonte, 1994). However, comparison of smoking smokers to nonsmokers and deprived smokers does allow at least a partial assessment of direct effects of tobacco versus effects due to withdrawal. Furthermore, analyses of CO levels confirmed smoking status differences between groups. In this case, pre-drug baseline data are not needed to establish withdrawal effects. Of course, use of a nonsmoking control group is limited by the possibility that smokers versus nonsmokers possess preexisting differences resulting in self-selection of smoking status. Nevertheless, these findings suggest an important direction for future research on visual-cognitive effects and mechanisms of action of nicotine and tobacco.
3. Enns and Di Lollo (1997) also included a -300-msec condition. This condition was excluded from the present study for three reasons. First, it shortened the block length. Second, the results of Enns and Di Lollo suggest that the condition would not provide meaningful data with regard to the questions of interest. Third, sudden onsets can compel eye movements despite instructions to maintain fixation (Theeuwes, Kramer, Hahn, \& Irwin, 1998) and $300 \mathrm{msec}$ is sufficient time to program and execute such an eye movement, meaning that in this condition the fixation would not be maintained on a significant proportion of trials.

(Manuscript received September 20, 2000; revision accepted for publication May 15, 2001.) 